\title{
Erratum: Multistep-capture-and-loss model for stabilization processes of hollow Ar atoms formed in a solid \\ [Phys. Rev. A 58, 5004 (1998)]
}

\author{
Keiko Suto and Takashi Kagawa \\ Department of Physics, Nara Women's University, Nara 630-8506, Japan
}

(Published 13 December 2000)

DOI: 10.1103/PhysRevA.63.019903 PACS number(s): 34.50.Dy, 32.30. $-\mathrm{r}, 32.70 .-\mathrm{n}, 32.90 .+\mathrm{a}, 99.10 .+\mathrm{g}$

The simulated results for the $K$ x-ray satellite spectra in hollow Ar atoms in the article should be obtained without an extra multiplication factor of the fluorescence yield of $\omega_{L \rightarrow K}$ in Eq. (4). So Eq. (4) and lines between the top and that just above Eq. (5) in the right column on page 5005 should be replaced by the following:

“ . . time-integrated population for the state. The intensity $I_{k}$ for the $k$ th $K$ x-ray satellite line is given by

$$
I_{k}=\Gamma_{L \rightarrow K}^{r} \int_{0}^{\infty} N_{k}(t) d t,
$$

where $\Gamma_{L \rightarrow K}^{r}$ is the radiative transition rate for the $L \rightarrow K E 1$ transition. In general the radiative transition rate for the $X$ $\rightarrow Y \quad E 1$ transition is expressed as ...',

Consequently, Fig. 2 should be replaced by the figure given here. There are some differences between the present simulated spectra obtained without $\omega_{L \rightarrow K}$ and the previous one, such as small changes of relative intensities of the $K$ x-ray satellite lines, the value of the magnitude of the intensity, and the value of $n_{M}$ for the strongest line among each group of satellite lines shown in the figure. However, these differences do not lead to serious discrepancies between the simulated and the experimental spectra. Therefore this change does not alter any of the physical considerations and conclusions of the article.

We thank Dr. Moribayashi of the Japan Atomic Energy Research Institute (JAERI) for pointing out this error.

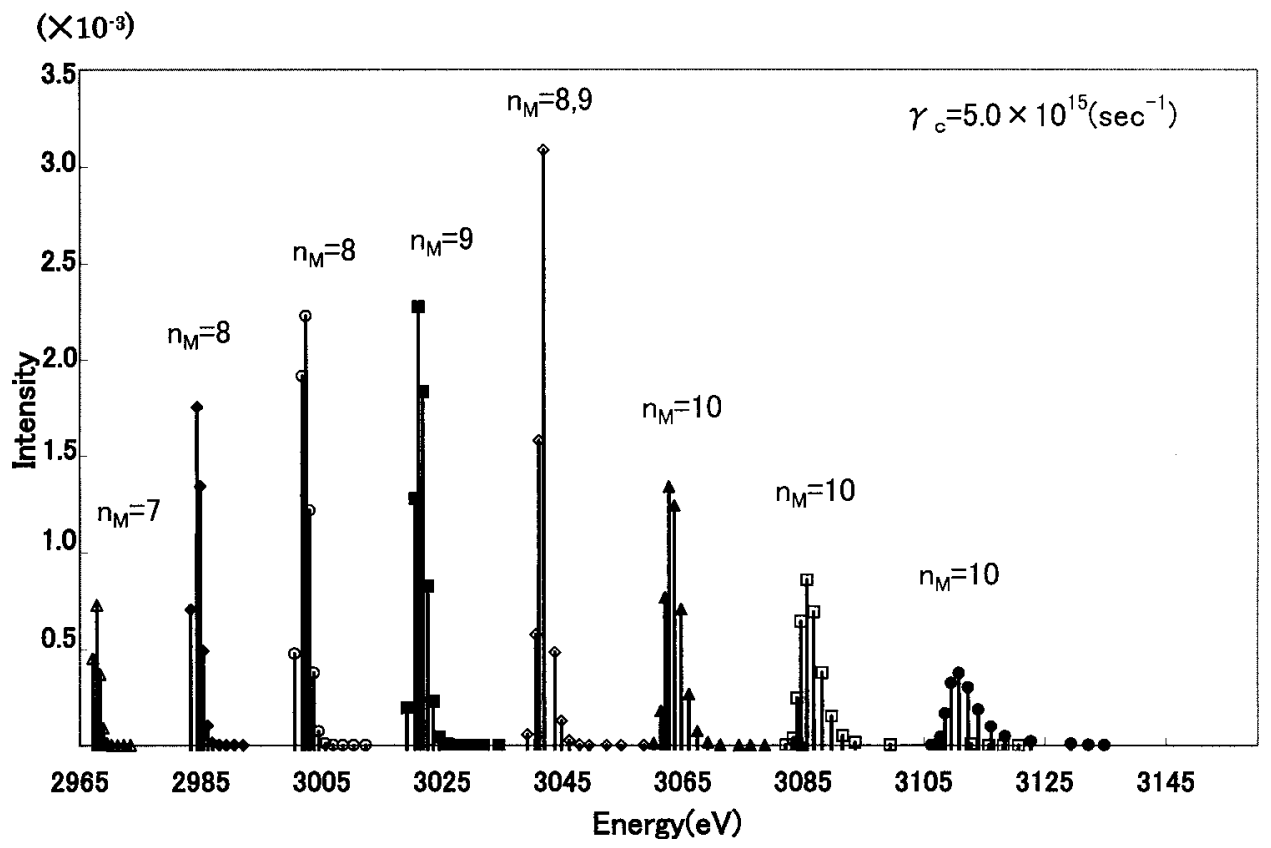

FIG. 2. Theoretical $K$ satellite spectra emitted from Ar hollow ions obtained with the MSCL model, and DFS transition energies starting from a $K M^{4}$ state whose population is unity at $t=0$. The value of the average rate $\gamma^{c}$ for the electron capture, and the number of $M$-shell spectator electrons $n_{M}$ in the configuration required for a state to give the largest intensity among lines in each group of satellite spectra, are given. The same symbols used in Fig. 1 to indicate each $K L^{n_{L}}$ satellite line are used in this figure. 